Journal of Environmental
ISSN: $2525-815 \mathrm{X}$

\title{
Gamificação como ferramenta de apoio no ensino de práticas na Educação Ambiental
}

\section{Gamification as a support tool in the teaching of practices in Environmental Education}

\author{
Armando Dias Duarte ${ }^{a}$, Deysianne Cristina Santos da Silva ${ }^{\mathrm{b}}$, José César Cavalcante dos Santos ${ }^{\mathrm{c}}$, \\ Emerson Philipe Sinesio ${ }^{\mathrm{b}}$, Flávio José Cordeiro de Andrade Filho ${ }^{\mathrm{c}}$ \\ ${ }^{a}$ Universidade Federal de Pernambuco-UFPE, Departamento de Recursos Hídricos. Av. Prof. Moraes Rego, n. 1235, \\ Cidade Universitária, Recife-PE. CEP: 50670-901.E-mail: armando01.dias@ gmail.com. \\ ${ }^{\mathrm{b}}$ UFPE, Centro Acadêmico do Agreste. Av. Campina Grande, s/n, Km 59, Nova Caruaru, Caruaru, Pernambuco. CEP: \\ 55014-9001. E-mail: deysianne.cristina22@ hotmail.com, emersonsinesio95@gmail.com. \\ ${ }^{c}$ Autarquia Educacional do Belo Jardim-AEB/FBJ. PE-166, s/n, BR, Belo Jardim, Pernambuco. CEP: 55150-000. E- \\ mail: cesarcavalcante16@ hotmail.com, flavioandradeeb@gmail.com.
}

A R T I C L E I N F O

Recebido 07 Mar 2020

Aceito 26 Nov 2020

Publicado 08 Dez 2020

\begin{abstract}
A B S T R A C T
One of today's great challenges is minimizing environmental impacts, which is related to changing habits. It raises the discussions of pedagogical methodologies and practices, together with Environmental Education, which is presented as a tool to support public policies, proposing different thoughts about environmental conservation. Holding attention and engaging those involved throughout the learning process can become one of the obstacles. Gamification then emerges as a teaching strategy to insert students into experiences in the themes addressed. This study aims to apply the gamification tool to support pedagogical practices, during environmental management in a Higher Education Institution located in the city of Belo Jardim - PE. For the development of the research, two questionnaires were used to verify students' environmental perception regarding issues related to environmental management and the second about gamification. In possession of the results, it was found that environmental issues of non-renewable energies and waste electrical and electronic equipment need more attention and that a tool for gamification together with pedagogical practices, presents itself as a form of a class engagement strategy, whose participation becomes more active according to the didactic preference adopted.
\end{abstract}

Keywords: Engagement, habits, games.

\begin{abstract}
R E S U M O
Um dos grandes desafios da atualidade, no sentido de minimizar os impactos ambientais, está relacionada na mudança de hábitos. O que eleva as discussões de metodologias e práticas pedagógicas, em conjunto com a Educação Ambiental que é apresentada como uma ferramenta de suporte as políticas públicas, propondo diferentes pensamentos acerca da conservação do meio ambiente. Prender a atenção e engajar os envolvidos durante todo o processo de aprendizagem, pode se tornar um dos obstáculos. Surge então a gamificação como estratégia de ensino, de forma a inserir os alunos, em vivências nas temáticas abordadas. $\mathrm{O}$ presente estudo objetivou aplicar a ferramenta de apoio às práticas pedagógicas, gamificação, durante a disciplina de gestão ambiental em uma Instituição de Ensino Superior localizada na cidade do Belo Jardim - PE. Para o desenvolvimento da pesquisa, foram utilizados dois questionários, o primeiro para verificar a percepção ambiental dos discentes a respeito de assuntos ligados a gestão ambiental e o segundo sobre gamificação. De posse dos resultados, foram constatados que os assuntos ambientais de energias não renováveis e resíduos de equipamentos eletroeletrônicos precisam de uma maior atenção e que ferramenta da gamificação em conjunto das
\end{abstract}


práticas pedagógicas, se apresenta como uma forma de estratégia de engajamento da turma, cuja participação se torna mais ativa de acordo com a preferência didática adotada.

Palavras-Chave: Engajamento, hábitos, jogos.

\section{Introdução}

Diante da reação negativa do meio ambiente, causada por práticas insustentáveis de consumo, há muito observa-se um resultado desproporcional das ações de preservação dos recursos naturais, o que acarreta um cenário desestruturado das políticas públicas e privadas voltadas para a sustentabilidade.

Em 2015 foi realizada uma conferência na cidade de Nova York, onde foram reunidos 193 estados-membros da ONU para discutir os objetivos do milênio, resultando na Agenda 2030. Esta agenda desafia os países no estabelecimento de práticas inovadoras para o alcance dos objetivos propostos, envolvendo, além das instituições governamentais, as organizações privadas no cotidiano dos brasileiros (PNUD, 2018). Dentre as metas estabelecidas, a erradicação da pobreza, o uso consciente da água, a eficiência energética e a educação de qualidade se destacam na pauta para a promoção do desenvolvimento sustentável global. Esta última sugere a garantia de capacitação dos indivíduos em todos os níveis da educação nos objetivos do desenvolvimento sustentável (PNUD, 2018). Apesar das pesquisas publicadas na plataforma Scielo, sobre a Gestão Ambiental, serem relacionadas, de maneira geral, às temáticas, como o aumento da produção e gestão da qualidade, nos últimas anos, estudos científicos têm revelado um aumento significativo no enfoque multidisciplinar e interdisciplinar proposto por Barbieri (2004), Barbieri \& Silva (2011) e Miranda, Miranda \& Ravaglia (2017), expondo seus avanços dentre as diversas abordagens, bem como a qualidade, a quantidade e a atualidade com que esse tema é trabalhado (Billar et al., 2019).

Tais desafios sugerem a criação de novos conceitos e metodologias, agora como forma de educação focada para a gestão ambiental, onde Silva (2018) destaca a importância da participação das escolas nesse contexto, afunilando o paralelo teoria-prática na forma de ensinar aos discentes, como exemplo, o plantio de mudas e a coleta seletiva de resíduos sólidos através de mutirões, enfatizando o comprometimento da comunidade nestas ações.

Esta relação meio-ambiente e o desenvolvimento revela a importância da Educação Ambiental (EA) em cursos de graduação, cujo papel de construir conhecimento em conexão com as necessidades da sociedade, faz com que os discentes lidem diretamente com questões ambientais, sociais e biológicas. Entretanto, sua abordagem pode alcançar maior eficácia quando tratada de forma interdisciplinar (Lima et al., 2019).

$\mathrm{Na}$ perspectiva de inserção de uma ferramenta de intervenção, o Conselho Nacional de Educação (CNE) aprovou a Resolução $\mathrm{n}^{\circ}$ 02/2012, e respectivo parecer, $n^{\circ} 14 / 2012$, de 05 de junho de 2012, o qual orienta a implantação da EA no Ensino Básico e Superior; nesse último, dando especial atenção aos cursos de formação de professores (Brasil, 2012). Nesse contexto, o papel das Instituições de Ensino (IE) é de extrema importância, incentivando futuros profissionais a atuarem no mercado de trabalho de forma ambientalmente correta (Nolasco, Tavares \& Bendassolli, 2006). Miranda, Miranda \& Ravaglia (2017) retomam esta abordagem destacando sua implicação, não só no envolvimento de todas as disciplinas, bem como inserindo a sociedade como um todo, em um enfoque interdisciplinar da EA. Esta surge como uma nova forma de alavancar os resultados das pesquisas e educação na formação de profissionais mais conscientes. Nesse interim, vale ressaltar a transcendência de se trabalhar o tema abordado, utilizando ferramentas e metodologias atuais. No ensino superior, Souza et al. (2019) ressaltam que a EA visa formar pesquisadores e discentes de qualidade para tentar minimizar os problemas ambientais. Dessa forma, busca-se maior efetivação quanto a implantação do tema abordado, a fim de fomentar e fortalecer, de forma quanti-qualitativa, o cuidado generalista com a educação ambiental.

No atual cenário de ensino, o professor se depara com o desafio de desenvolver estratégias que busquem, de forma eficaz, a motivação entre os discentes durante o processo de aprendizagem (Araújo \& Carvalho, 2017). Dentre as diversas abordagens de ensino e aprendizagem, a Gamificação/Gamification é apresentada como uma ferramenta de suporte às práticas pedagógicas, que podem ser presenciais ou através de plataformas eletrônicas, e que permite a sua inclusão no ensino fundamental, superior e treinamentos empresariais (Zainuddin et al., 2020). Permite, também, apoiar e motivar o usuário através do objetivo estabelecido, seja por comportamentos saudáveis ou participação de atividades de aprendizagem (Koivisto \& Hamari, 2019). 
Kapp (2012) define que a metodologia da Gamificação utiliza mecanismos de jogos, elementos de estética e pensamentos para envolver os discentes visando a resolução de problemas. Seus conceitos iniciais e aplicações surgiram por volta de 2010, com alguns estudos acadêmicos (Hamari, Koivisto \& Sarsa, 2014).

Diante do exposto, o presente estudo objetivou analisar a percepção ambiental por meio da aplicação da ferramenta da gamificação, nas práticas docentes da disciplina de gestão ambiental, em uma Instituição de Ensino Superior (IES).

\section{Material e Métodos}

A pesquisa foi realizada com uma turma do terceiro período do curso de Administração, totalizando 30 discentes, em uma IES localizada na cidade do Belo Jardim - PE. De forma a serem coletados os dados da pesquisa, foram elaborados dois questionários distintos, baseados nas premissas de Günther (2003). O primeiro questionário, com abordagem sobre a gestão ambiental, as perguntas foram elaboradas, baseadas nos conceitos apresentados pela disciplina, como, por exemplo, questões sobre a importância de ações que visam a melhoria dos recursos hídricos, dos resíduos sólidos, do consumo de energia não renovável, da geração de resíduos eletroeletrônicos e da educação ambiental. $\mathrm{O}$ segundo questionário abordou a identificação do perfil dos discentes quanto as práticas adotadas pela aplicação da gamificação.
Optou-se por utilizar uma escala likert de cinco pontos, que, de acordo com Zanon et al. (2013), compreende valores entre um (discordo totalmente) e cinco (concordo totalmente). Outros estudos utilizaram a mesma escala likert, com o objetivo se obter resultados para a avaliação das práticas da gamificação (Li, Grossman \& Fitzmaurice, 2012; De-Marcos et al., 2014; Lopes, Toda \& Brancher, 2015).

Posteriormente, de posse dos resultados obtidos com os questionários, estratégias pedagógicas foram adotadas, com aplicação de práticas de gamificação em sala de aula.

\section{Resultados e Discussão}

Os entrevistados foram $63 \%$ do sexo feminino, com uma média de idade de 25 anos. considerando as respostas sobre os conceitos de gestão ambiental; 90\% afirmaram que ouviram falar no termo, porém, $50 \%$ não possuem o conceito claro. Indagados se praticam ações ambientais em casa, $85 \%$ afirmam que sim. Quanto à motivação das pessoas na realização de práticas ambientais, 38\% relacionam questões financeiras, $43 \%$ a preocupação com o meio ambiente e $19 \%$ as questões sociais. Os principais problemas observados, de acordo com os discentes, são: má gestão dos recursos hídricos (68\%) e má gestão sanitária (32\%).

O Quadro 1 apresenta as perguntas utilizadas no questionário e os resultados do ranking médio sobre as questões ambientais.

Quadro 1. Informações sobre a percepção da Gestão ambiental obtidas após aplicação de questionário com discentes do terceiro período do curso de Administração, em uma Instituição de Ensino Superior localizada na cidade do Belo Jardim, Pernambuco. Fonte: Duarte et al. (2020).

\begin{tabular}{|c|c|c|}
\hline Perguntas & Ranking médio & Grau de concordância \\
\hline $\begin{array}{c}1.1 \text { - Você concorda em ações que } \\
\text { visam a melhoria dos Recursos } \\
\text { Hídricos são fundamentais? }\end{array}$ & 4,75 & Concorda totalmente \\
\hline $\begin{array}{c}1.2 \text { - Você concorda em ações que } \\
\text { visam a melhoria dos Resíduos } \\
\text { Sólidos são fundamentais? }\end{array}$ & 4,62 & Concorda totalmente \\
\hline $\begin{array}{c}1.3 \text { - Você concorda em ações que } \\
\text { visam a redução do consumo de } \\
\text { energias não renováveis são } \\
\text { fundamentais? }\end{array}$ & 4,31 & Concorda parcialmente \\
\hline $\begin{array}{c}1.4 \text { - Você concorda em ações que } \\
\text { visam a redução de resíduos de } \\
\text { equipamentos eletroeletrônicos } \\
\text { são fundamentais? }\end{array}$ & 3,37 & Concorda totalmente \\
\hline $\begin{array}{c}1.5 \text { - Você concorda que a } \\
\text { Educação Ambiental é uma } \\
\text { ferramenta de grande importância, } \\
\text { para políticas ambientais? }\end{array}$ & 4,56 & Concorda parcialmente \\
\hline 1.6 - Você concorda que se faz & 3,81 & \\
\hline
\end{tabular}




\begin{tabular}{|c|c|c|}
\hline $\begin{array}{c}\text { necessário grandes investimentos } \\
\text { na área ambiental, para que as } \\
\text { políticas públicas sejam } \\
\text { alcançadas? }\end{array}$ & \\
\hline $\begin{array}{c}\text { 1.7 - Você concorda que para } \\
\text { tomar decisões ambientais, se faz } \\
\text { necessário um alto nível de } \\
\text { instrução? }\end{array}$ & 3,75 & Concorda parcialmente \\
\hline
\end{tabular}

De acordo com o que foi observado pelas respostas dos questionários ambientais, se faz necessário um melhor aproveitamento dos conteúdos sobre as questões do consumo de energias não renováveis (item 1.3) e do assunto relacionado com os resíduos de equipamentos eletroeletrônicos (item 1.4), pois apresentam graus de concordância inferiores aos demais temas abordados. Esse último assunto, tem levado a discussões de forma urgente no contexto brasileiro, de forma a ser enfatizado em escala global (Oliveira Neto, Silva \& Machado Santos, 2019). No item 1.5, sobre a EA ser uma ferramenta de suporte às políticas ambientais, os discentes concordam totalmente, concordam parcialmente sobre se faz necessário grandes investimentos na área ambiental, o que leva a crer que, de acordo com a percepção da turma, a EA pode ser adotada como estratégia de melhoria das políticas públicas, sem muitos custos financeiros. Como afirma Monteiro (2020), que a relação entre discentes e docentes, contribui na formação de cidadãos mais conscientes e que repercute na melhoria da qualidade de vida nas cidades e no desenvolvimento ambiental e social das mesmas.

Os discentes, quando perguntados se ouviram falar do termo gamificação, $81 \%$ responderam que nunca ouviram falar. Sobre a preferência de forma a ser trabalhada, $81 \%$ afirmam que preferem trabalhar em equipe. considerando os fatores que levam as pessoas a se sentirem atraídas por jogos, $57 \%$ responderam que é o desafio, $32 \%$ diversão, $11 \%$ competição e nenhuma resposta para o quesito autonomia.

$\mathrm{Na}$ opção relativa à preferência de recursos de aprendizagem, $25 \%$ preferem figuras, diagramas e outros recursos visuais, $19 \%$ preferem receber instruções verbais e apenas escutar para aprender e, 56\% afirmam que aprendem melhor realizando as tarefas de forma ativa. O Quadro 2 demonstra as perguntas utilizadas e o ranking médio das respostas do questionário sobre gamificação.

Quadro 2. Resumo das informações sobre gamificação obtidas da aplicação de questionário com discentes do terceiro período do curso de Administração, em uma Instituição de Ensino Superior localizada na cidade do Belo Jardim, Pernambuco. Fonte: Duarte et al. (2020).

\begin{tabular}{|c|c|c|}
\hline Perguntas & Ranking médio & Grau de concordância \\
\hline $\begin{array}{c}2.1 \text { - Você acha importante } \\
\text { estimular a competição entre } \\
\text { equipes através de uma pontuação } \\
\text { (score)? }\end{array}$ & 3,43 & Nem concorda, nem discorda \\
\hline $\begin{array}{c}2.2 \text { - Você acha importante o uso de } \\
\text { estudos de caso como desafios na } \\
\text { Gamificação? }\end{array}$ & 4,32 & Concorda parcialmente \\
\hline $\begin{array}{c}2.3 \text { - Você ficou motivado(a) a } \\
\text { participar das atividades } \\
\text { desenvolvidas? }\end{array}$ & 4,56 & Concorda parcialmente \\
\hline $\begin{array}{c}2.4 \text { - Você concorda que a } \\
\text { Gamificação estimula o trabalho em } \\
\text { equipe? }\end{array}$ & 3,62 & Concorda parcialmente \\
\hline $\begin{array}{c}2.5 \text { - Você concorda que o uso da } \\
\text { Gamificação torna a disciplina mais } \\
\text { atrativa? }\end{array}$ & 3,43 & Concorda totalmente \\
\hline $\begin{array}{c}2.6 \text { - Você considera que a } \\
\text { Gamificação pode ser uma } \\
\text { ferramenta de suporte ao ensino? }\end{array}$ & 4,62 & Concorda totalmente \\
\hline $\begin{array}{c}2.7 \text { - Você considera que no ensino, } \\
\text { novas tecnologias são necessárias }\end{array}$ & 4,62 & \\
\hline
\end{tabular}




\begin{tabular}{|c|c|c|}
\hline para atrair atenção? & & \\
\hline $\begin{array}{c}2.8 \text { - A Gamificação deveria ser } \\
\text { aplicada em outras disciplinas? }\end{array}$ & 4,37 & Concorda parcialmente \\
\hline $\begin{array}{c}2.9 \text { - Você considera que os } \\
\text { assuntos abordados em sala de aula, } \\
\text { após a gamificação, servirão para o } \\
\text { futuro profissional? }\end{array}$ & 4,68 & Concorda totalmente \\
\hline
\end{tabular}

A partir dos resultados obtidos com o questionário sobre a gamificação, no item 2.7 , os discentes concordam totalmente de que novas tecnologias são necessárias para atrair atenção, contudo Barreto, Becker \& Ghisleni (2019), explicam que o uso da gamificação aliada à tecnologia nas atividades de ensino, podem ser optativas, e que os docentes podem adotar dinâmicas similares aos jogos, portanto, a estratégia adotada pelo docente foi a criação de grupos, onde os discentes passam a assumir papéis de consultores ambientais, visto que 56\% afirmam que aprendem de forma ativa. A cada aula realizada, o assunto foi abordado e ao final da aula teórica, foram realizados estudos de caso, cujos grupos procuram solucionar as problemáticas, através de discussões e outras ferramentas desenvolvidas no decorrer da aula. Essa forma de inserção dos discentes é demonstrada no estudo realizado por Buckley, Garvey \& McGrath (2011) como satisfatória, cujo incentivo em um cenário dinâmico melhorou as habilidades quanto à tomada de decisão. De forma a pontuar as equipes, o docente então realizou a avaliação de cada grupo, utilizando fichas com uma escala de 1 a 5. Dessa forma, os grupos passam a ganhar conquistas a cada atividade realizada, o que contribuiu para o aumento do engajamento da turma, como constatados em estudos realizados, que demonstraram que o ganho de conquistas, proporcionam uma maior participação nas atividades gamificadas (McDaniel, Lindgren \& Friskics, 2012; Li, Grossman \& Fitzmaurice, 2012).

De forma a verificar a aplicação da gamificação em sala de aula, Barata et al. (2013) realizaram uma pesquisa de satisfação, utilizando questionários com escala likert de 1 a 5 , entre os discentes dos cursos de mestrado em Sistemas de Informação e Engenharia computacional, e constataram que os discentes concordam (4) que a gamificação deveria se estender entre outros cursos, o que corrobora com o resultado encontrado na pesquisa (item 2.8) de que a gamificação deveria ser aplicada em outras disciplinas. Barata et al. (2013) também relatam que os desafios propostos, modelados para a disciplina permitiram aos discentes, serem mais criativos na resolução das problemáticas. Assim,
Barreto, Becker \& Ghisleni (2019) também concluem que a gamificação no ensino é uma prática estimulante e motivadora que se adequa às capacidades de cada discente, conforme verificado nas respostas obtidas no item 2.3 e que a gamificação é um campo promissor nas áreas da saúde, ambiente, governo e na educação, verificado através do item 2.6, cujos discentes concordam totalmente de que a prática pode ser uma ferramenta de suporte ao ensino.

\section{Conclusão}

A ferramenta da gamificação, aliada às práticas pedagógicas da disciplina de gestão ambiental, se apresenta como uma forma de estratégia de engajamento dos discentes, cuja participação se torna mais ativa, de acordo com a preferência adotada pela forma de aprendizagem e as necessidades de conteúdo a ser estudado. Os conceitos são postos em prática através de exemplos, associados à imersão da turma com as problemáticas da gestão ambiental, por meio de atividades que contribuem para a formação de profissionais capazes de minimizar os problemas ambientais.

\section{Agradecimentos}

Agradecemos à Autarquia Municipal do Belo Jardim, pelo espaço proporcionado para a realização da pesquisa.

\section{Referências}

Araújo, I.; Carvalho, A. A. 2017. Capacitar professores para o uso da gamificação. Atas do XIX Simpósio Internacional de Informática Educativa e VIII Encontro do CIED-III Encontro Internacional, pp. 264269.

Barbieri, J. C. 2004. A educação ambiental e a gestão ambiental em cursos de graduação em administração: objetivos, desafios e propostas. Revista de Administração Pública, 38, (6), 919-946.

Barbieri, J. C.; Silva, D. D. 2011. Desenvolvimento sustentável e educação ambiental: uma trajetória comum com muitos desafios. RAM. Revista de Administração Mackenzie, 12, (3), 51-82. 
Barata, G.; Gama, S.; Jorge, J. A. P.; Gonçalves, D. 2013. Engagind Enginnering Students with Gamification. Proceedings of the fifth outing of the International Conference on Games and Virtual Worlds for Serious Applications. Doi: $\quad 10.1109 /$ vsgames.2013.6624228.

Brasil. Resolução CNE/CP 2, de 15 de junho de 2012, e respectivo parecer $n^{\circ} 14 / 2012$, de 05 de junho de 2012. Estabelece as Diretrizes Curriculares Nacionais para a Educação Ambiental, bem como o parecer correspondente, $\mathrm{n}^{\circ}$ 14/2012. Diário Oficial da União, Brasília 15 jun. 2012. Seção 1, 18.

Bilar, A. B. C.; Silva, A. H. G.; Silva, A. C.; Silva, C. M.; Souza, E. K.; Santos, I. B.; Albuquerque, S. 2019. Gestão ambiental em publicações científicas nacionais: uma revisão sistemática. Journal of Environmental Analysis and Progress, 4, (4), 290-296.

Buckley, P.; Garvey, J.; McGrath, F. 2011. A case study on using prediction markets as a rich environment for active learning. Computers \& Education, 56, (2), 418-428. Doi: 10.1016/j.compedu.2010.09.001.

Barreto, C. H. da C.; Becker, E. L. S.; Ghisleni, T. S. 2019. Gamificação: uma prática da educação 3.0. Research Society and Development, 8, (4). Doi: 10.33448/rsdv8i4.942.

De-Marcos, L.; Domínguez, A.; Saenz-deNavarrete, J.; Pagés, C. 2010. An empirical study comparing gamification and social networking on e-learning. Computers \& Education, 75, 2013, 82-91. Doi: 10.1016/j.compedu.2014.01.012.

Miranda, F. H. da F.; Miranda, J. A.; Ravaglia, R. 2017. Abordagem Interdisciplinar em Educação Ambiental. Revista Práxis, 2, (4), 1-6.

Oliveira Neto, J. F.; Silva, M. M.; Santos, S. M. 2019. A Mini-Review of E-Waste Management in Brazil: Perspectives and Challenges. CLEAN-Soil, Air, Water, 47, (9), 1900152.

Souza, I. P.; Chaves, L. S. S.; Saraiva, L. C. D. S. A.; Santos, M. R.; Mendonça, M. S. 2019. Educação ambiental e seus desafios no ensino superior: Um Estudo de caso do Instituto Federal de Educação, Ciência e Tecnologia do Pará, campus Bragança. Ambiente \& Educação-Revista de Educação Ambiental, 24, (1), 32-53.

Günther, H. 2003. Como Elaborar um Questionário/ Série: Planejamento de Pesquisa nas Ciências Sociais, (1),
Laboratório de Psicologia Ambiental Instituto de Psicologia - Universidade de Brasília.

Hamari, J.; Koivisto, J.; Sarsa, H. 2014. Does Gamification Work? - A Literature Review of Empirical Studies on Gamification. 2014 47th Hawaii International Conference on System Sciences. Doi: 10.1109/hicss.2014.377.

Kapp. K. M. 2012. The Gamification of Learning and Instruction: Game-based methods and strategies for training and education. San Francisco: Pfeiffer.

Koivisto, J.; Hamari, J. 2019. The rise of motivation al information systems: A review of gamification research. International Journal of Information Management, 45, 191-210.

Doi: 10.1016/j.ijinfomgt.2018.10.013.

Li, W.; Grossman, T.; Fitzmaurice, G. 2012. GamiCAD: a gamified tutorial system for first time autocad users. In: Proceedings of the 25th annual ACM symposium on User interface software and technology, pp. 103112.

Lima, A. A.; Bakke, I. A.; Melo, A. C.; Lima, L. V. S.; Freitas, A. L.; Martins, A. P. F. 2019. Representações sociais em educação ambiental com estudantes de três cursos da UFCG-Campus de Patos. Revista Agropecuária Científica no Semiárido, 14, (2), 129-140.

Lopes, R. A.; Toda, A. M.; Brancher, J. D. 2015. Um estudo preliminar sobre elementos extrínsecos e intrínsecos do processo de Gamification. Revista Brasileira de Informática na Educação, 23, (3), 164-173.

McDaniel, R.; Lindgren, R.; Friskics, J. 2012. Using badges for shaping interactions in online learning environments. In 2012 IEEE International Professional Communication Conference, IPCC 2012 [6408619] (IEEE International Professional Communication Conference).

Doi: 10.1109/IPCC.2012.6408619.

Monteiro, A. R. 2020. Educação ambiental: um itinerário para a preservação do meio ambiente e a qualidade de vida nas cidades. Revista de Direito da Cidade, 12, (1), 830850. Doi: 10.12957/rdc.2020.42078.

Nolasco, F. R.; Tavares, G. A.; Bendassolli, J. A. 2006. Implantação de programas de gerenciamento de resíduos químicos laboratoriais em universidades: análise crítica e recomendações. Engenharia Sanitária e Ambiental, 11, (2), 118-124. 
PNUD. Plataforma Agenda 2030, 2018. Disponível em: http://www.agenda2030.com.br/sobre/. Acesso em: 06 mar 2020.

Silva, J. D. N. 2018. Sustentabilidade ambiental na educação profissional: desafios para o desenvolvimento sustentável na Baixada Maranhense. Revista Brasileira de Educação Ambiental, 13, (3), 275-283.
Zainuddin, Z.; Wah Chu, S. K.; Shujahat, M.; Perera, C. J. 2020. The impact of gamification on learning and instruction: A systematic review of empirical evidence. Journal of Educational Research Review, 100326. Doi: 10.1016/j.edurev.2020.100326.

Zanon, C.; Bastianello, M. R.; Pacico, J. C.; Hutz, C. S. 2013. Desenvolvimento e validação de uma escala de afetos positivos e negativos. Revista Psico-USF, 18, (2), 193-201. 